Review

\title{
Analysis of Migraine Pathophysiology by Magnetic Resonance Imaging
}

Yasushi Shibata ${ }^{1,}{ }^{*}$, Masayuki Goto ${ }^{2}$, Sumire Ishiyama ${ }^{3}$

1. Department of Neurosurgery, Headache Clinic, Mito Medical Center, Mito Kyodo General Hospital, University of Tsukuba, Ibaraki, Japan; E-Mail: yshibata@md.tsukuba.ac.jp

2. Degree Programs in Medical Sciences, Graduate School of Comprehensive Human Sciences, University of Tsukuba, Ibaraki, Japan; E-Mail: right.happy.50@gmail.com

3. Faculty of Health Sciences, Tsukuba University of Technology, Tsukuba, Ibaraki, Japan; E-Mail: suishiyama@ad.tsukuba-tech.ac.jp

* Correspondence: Yasushi Shibata; E-Mail: yshibata@md.tsukuba.ac.jp

Academic Editor: Guodong Cao

Special Issue: The Pathophysiology and Treatment for Migraine

OBM Neurobiology

2022, volume 6, issue 1

doi:10.21926/obm.neurobiol.2201115
Received: October 25, 2021

Accepted: January 28, 2022

Published: February 08, 2022

\begin{abstract}
Magnetic resonance imaging (MRI) has been used to investigate migraine pathophysiology because it is a non-invasive technique. The main aim of clinical imaging for patients with headaches is to exclude secondary headaches due to organic lesions. Conventional structural imaging techniques such as routine MRI demonstrate white matter lesions, changes in gray matter volume or cortical thickness, and cerebral blood flow in patients with migraine. Changes in metabolite levels are observed by magnetic resonance spectroscopy. Diffusion tensor imaging, neurite orientation dispersion, density imaging, and functional MRI reveal dynamic real-time functional changes in brain microstructures. These analyses have been applied not only for comparing patients with migraine and healthy controls but also for understanding the dynamic changes in brain function during the cyclic migraine ictal phase. Although these analyses have demonstrated migraine pathophysiology, there are still many limitations. Following the improvement in imaging technology, further research on this topic is in progress.
\end{abstract}

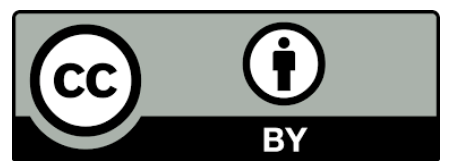

(C) 2022 by the author. This is an open access article distributed under the conditions of the Creative Commons by Attribution License, which permits unrestricted use, distribution, and reproduction in any medium or format, provided the original work is correctly cited. 


\section{Keywords}

Migraine; MRI; diffusion tensor imaging; functional MRI

\section{Introduction}

Migraine is diagnosed based on clinical symptoms and history alone. The main aim of clinical imaging is to exclude secondary headaches due to organic lesions. However, because the pathophysiology of migraine is not yet fully clear, many imaging studies have been conducted. Magnetic resonance imaging (MRI), in particular, has been used intensively as an imaging method for research because it is a non-invasive technique and does not involve radiation exposure. The present review aims to provide past and recent clinical studies involving the use of various MRI techniques for diagnosing patients with migraine. We have selected important studies that used clinically available MRI methods.

\section{White Matter Lesions}

Conventional diagnostic MRI is used to detect structural abnormalities in migraine imaging studies. Standard T2-weighted images are used to detect white matter hyperintensity (WMH). Although WMH is common and progressive in patients with migraine [1], it remains unclear whether WMH in migraine is a biomarker of headache susceptibility or a by-product of migraine, and further studies are required to clarify this aspect [2].

\section{Gray Matter Volume and Cortical Thickness}

T1-weighted images are used to analyze gray matter volume. In patients with migraine, the amount of gray matter in the brainstem is increased, which supports the theory that the brainstem plays a major role in the pathophysiology of migraine [3]. On the contrary, an increased amount of gray matter in the temporal gyrus and fusiform gyrus is observed only in children, which may be related to the unique aspects of migraine in children [3]. Moreover, compared to males, females have thicker cortices in nociception-related areas such as the primary somatosensory cortex, precuneus, supplementary motor area, basal ganglia, and amygdala [4]. In particular, the precuneus is thicker in patients with migraine. The precuneus is the hub of major resting-state functional connectivity, and its connectivity is $14 \%$ higher in females than in males. A potential function of the precuneus is to actively suppress migraine-induced arousal in some cortical areas, which leads to cortical deactivation in other cortical areas [5]. This finding suggests that a network of brain regions, including the precuneus, may play a role in sex differences in migraine [6].

\section{Cerebral Blood Vessels}

It is hypothesized that vasodilation of the external carotid artery system serves as a trigger for migraine without aura, and magnetic resonance angiography (MRA) measurements of the diameter of intracranial and extracranial blood vessels were performed to test this hypothesis. The results revealed no difference in the diameter of vessels in the external carotid artery system (middle 
meningeal artery and superficial temporal artery) during headache attacks and non-attacks, while the internal carotid artery system (intracranial and extracranial internal carotid artery, and middle cerebral artery) was significantly vasodilated [7].

It has also been reported that microhemorrhage on the tent and simultaneous existence of microhemorrhage and ischemia are significantly more common in migraine without aura in the elderly, which supports the hypothesis that microvessels are involved in the pathogenesis of migraine [8].

A prospective cohort study of women aged 25 to 42 years with migraine with a 20-year followup suggested that migraine is a potential risk factor for major cardiovascular events. The mechanisms by which migraine increases the risk of cardiovascular events include increased thrombotic susceptibility, common genetic markers, and inflammatory processes [9].

A cohort study that investigated the association between migraine and subcortical cerebrovascular lesions within a racially and ethnically diverse population included 546 imaging studies and showed that migraine doubled the odds ratio for the occurrence of asymptomatic stroke [10].

\section{Perfusion Imaging}

Perfusion imaging shows and quantifies the perfusion state of a tissue-based on cerebral blood flow (the amount of blood passing through the capillary beds of a tissue, quantified as $\mathrm{mL} / 100 \mathrm{~g}$ tissue per minute), cerebral blood volume (the average amount of blood in the tissue bed, quantified as $\mathrm{mL} / 100 \mathrm{~g}$ tissue), and mean transit time (the time taken for blood to pass through the tissue). Cerebral perfusion has been investigated in a case study of hemiplegic migraine, a rare form of migraine associated with loss of motor function [11]. In a retrospective study using arterial spin labeling (ASL) and MRA, pediatric migraineurs with aura showed ipsilateral hypoperfusion [12]. In addition, pediatric patients with migraine who had perfusion changes during migraine were more likely to have symptoms such as aura, movement disorders, confusion, and hospitalization than those who did not have such changes, although the differences in the timing of MRI between cases may have affected the results [13]. Differences in the aura, presence of drug abuse, and types of migraine such as familial migraine or atypical migraine may also affect perfusion imaging findings.

\section{Magnetic Resonance Spectroscopy}

Magnetic resonance spectroscopy (MRS) is a method of measuring the concentration of local metabolites in tissues. Metabolites provide information on energy utilization, white matter (WM) degradation, and neurotransmitter activity, and these measurements can provide various insights into brain structure, function, and metabolism. A study measuring brain metabolites in pediatric patients with migraine showed decreased phosphocreatine levels and higher cytoplasmic $\mathrm{pH}$ in the occipital lobe, along with higher relative oxidation rates in the mitochondria [14]. Decreased phosphocreatine concentrations in the occipital lobe have also been detected in adult migraineurs, and these results indicate the possibility of mitochondrial dysfunction, i.e., brain metabolic disorders, in migraineurs [15].

A previous study suggested that decreased magnesium levels in adult migraineurs are an independent risk factor for migraine attacks [16], and such a decrease has been reported in the occipital, frontal, and temporal lobes of migraineurs [17]. Magnesium may play a role in the 
pathogenesis of migraine through its ability to inhibit vasoconstriction and platelet aggregation and stabilize cell membranes [18]. Magnesium is also involved in the regulation of vascular tone and responsiveness to endogenous hormones and neurotransmitters through its relationship with $\mathrm{N}$ methyl-d-aspartic acid (NMDA) receptors [19]. In pediatric patients with migraine, oral administration of magnesium has been shown to decrease the number and intensity of headache days [20]; thus, suggesting that the administration of magnesium as a preventive therapy may be particularly effective in children.

\section{Diffusion Tensor Imaging}

Each molecule has Brownian motion. This motion is more limited in water than in air and more limited in tissue than in water. In diffusion-weighted imaging (DWI), mutually perpendicular three motion probing gradients (MPGs) are applied. The signal intensity of the molecule changes parallelly with the decrease in MPG. Thus, DWI can measure the mean diffusivity (MD) of water in the region of interest. Acute cerebral infarction, intense cellular brain tumor, and cerebral abscess have limited diffusion, which is noted as high intensity in DWI.

To measure the direction in addition to the amount of diffusion, more than 6 MPGs is required, and the image can be acquired by diffusion tensor imaging. In neural tissues, neurites such as axon and dendrite limit molecular diffusion. In the two-dimensional space, the direction and amount of diffusion are expressed as a vector. In the three-dimensional space, the direction and amount of diffusion are expressed as a tensor and ellipsoid, respectively. The amount of longitudinal diffusion and perpendicular diffusion is expressed as axial diffusivity (AD), and radial diffusivity (RD), respectively, and the mean value of three mutually perpendicular diffusivities is MD (Figure 1). Fractional anisotropy (FA) is defined as the index of directionality of diffusion. In free water, water molecules freely diffuse into every direction; hence, FA is defined as 0 . If the diffusion is restricted to only one direction, FA becomes 1 . In the central nervous system, homogenously directed neural fibers such as the corpus callosum and internal capsule have high FA values; hence, these regions are good targets for investigation with DTI.

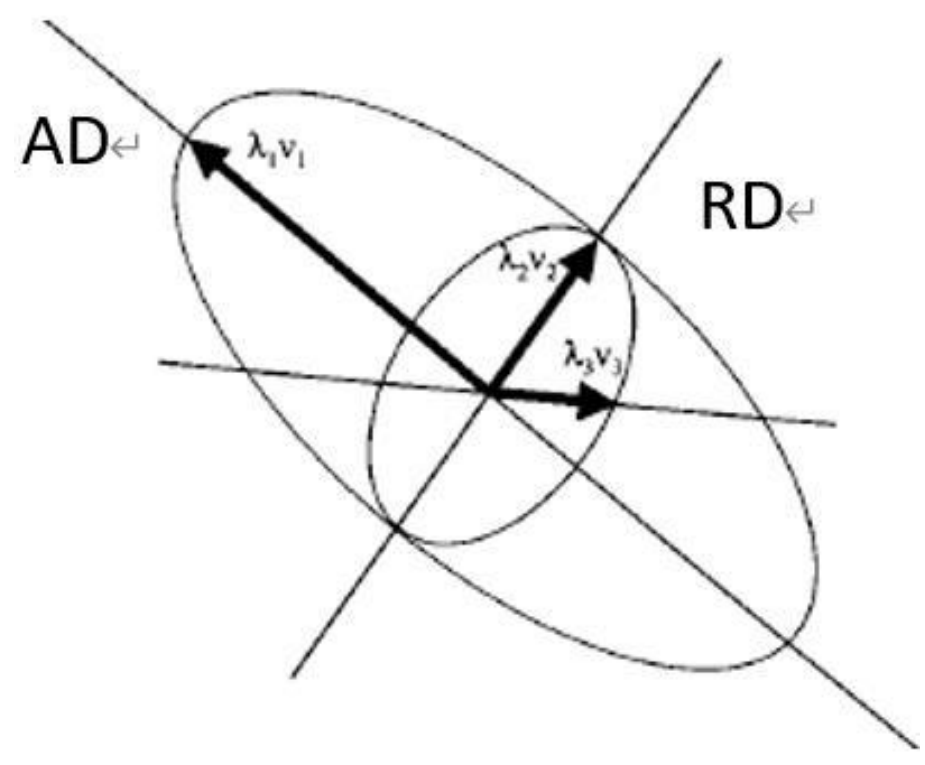

Figure 1 Parameters of DTI. 


\section{DTI Analysis for Patients with Migraine}

DTI has been used for research in migraine pathophysiology since 2006 [21]. In this period, MD and FA were measured as one mean value of the whole brain. Consequently, migraine-specific pathology such as pain matrix-specific abnormality was not revealed. Whole-brain analysis requires a huge data load, resulting in a longer calculation time. To solve these issues, tract-based spatial statistics (TBSS) was developed, which became the standard method for whole-brain analysis with DTI. In TBSS, whole-brain DTI data were consolidated into WM skeleton, and this skeleton alone was analyzed. By using TBSS, FA decrease at the corpus callosum and right frontal WM were detected in patients with migraine [22-24].

The image acquisition of DTI is performed using a 3.0 T MRI machine because image resolution is better for a high-intensity magnetic field. We investigated DTI for patients with migraines by using a 1.5 T MRI machine that has been widely used in clinical situations [25]. The presence of aura and medication overuse headache was also simultaneously investigated. In patients with migraine, RD was increased at WM around the corpus callosum, especially for patients with medication overuse headache (Figure 2). MD also showed the same results. FA was decreased around the corpus callosum for patients with migraine with aura and medication overuse headache (Figure 2).

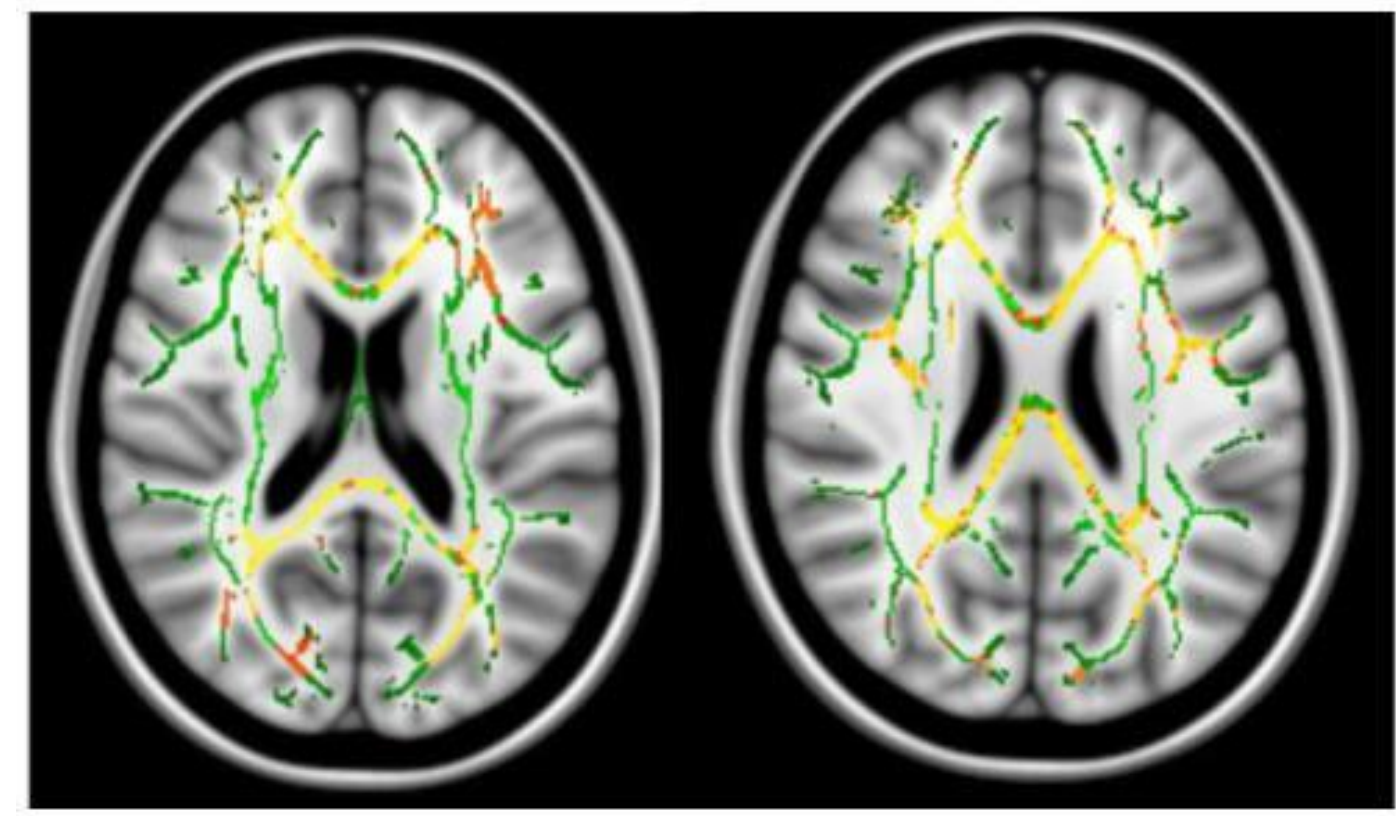

Figure 2 DTI results for a patient with medication overuse headache. The normal skeleton is shown in green, and the statistically abnormal skeleton is shown as yellow and red.

Many studies have discussed whether these abnormalities of diffusion parameters are causes or results of migraine. Some research studies have indicated the relationship between the frequency of migraine attacks and FA decrease, but other studies have not reported similar findings. Most reported studies were cross-sectional studies that showed the parameters at the measured time points. To investigate serial changes in parameters, a long-term longitudinal study is required. Migraine is not a stable disease. With the progression in migraine pathology, continuous fluctuations in diffusion parameters are suggested to occur [22]. The authors showed that axonal 
demyelination and WM damage contribute to the transient increase in both RD and $A D$, a decrease in FA in the episodic migraine phase, and a decrease in RD and AD with an increase in FA in the chronic migraine phase.

Migraine attacks may contribute to axonal damage; however, neural tissues exhibit plasticity. Future studies should reveal the quality and quantity of axonal damage induced by migraine attack and spontaneous repair.

Migraine is a periodic and episodic disease; therefore, for comparing migraine patients with healthy controls, we must consider image acquisition time for migraine attacks. In most research studies, images were acquired in the inter-ictal period. DTI analysis during migraine attacks has been rarely reported. In a research study on imaging for 24 patients with migraine in the ictal and interictal period, thalamic FA was higher than that of healthy volunteers in the inter-ictal period and decreased in the ictal period [26]. In this study, the patients who underwent imaging in the ictal period and the inter-ictal period were different persons. This study did not acquire continuous images for the same patients.

\section{The Problem of DTI}

In the corpus callosum or the pyramidal tract, neuro fibers run almost in the same direction. However, in other brain regions, neuro fibers run in all directions, which sometimes result in intersections at various angles. Because the mean value of each parameter in the voxel is calculated in DTI, the intersections are not appropriately evaluated in DTI. To solve these problems, various methods such as Q-space imaging, Q-ball imaging, diffusion kurtosis imaging, and diffusion spectrum imaging were developed and investigated [27]. These acquisition and analysis methods require a huge volume of data and longer analysis time; hence, the clinical application of these methods is limited in clinical settings.

The values of $F A, R D$, and $A D$ are influenced not only by axons but also by blood vessels, neural cell bodies, and glia. A high density of neurites leads to high FA, while the dispersion of neurites results in low FA. Hence, new analysis methods were developed to separately measure neurite density and dispersion.

\section{Neurite Orientation Dispersion and Density Imaging}

In DWI, molecules are considered to diffuse into every direction in the same amount. Hence, a round sphere is used as the model, and only the amount of diffusion is measured. Free water in the ventricle or subarachnoid space is determined with this model. In DTI, the ellipsoid with a particular volume is used as the model, and FA, AD, and RD are measured. Molecules in neural cells or glial cells are determined with this model. Neurites such as axon and dendrite are modeled as a stick, and neurite density and dispersion are measured in Neurite Orientation Dispersion and Density Imaging (NODDI) [28]. NODDI requires a long calculation time; however, the acquisition is almost the same as that obtained with DTI. TBSS is used for whole-brain analysis in the same manner as DTI. The pathophysiological analysis of migraines using NODDI has not yet been reported, and our research group is working on this topic. 


\section{Clinical Study of Functional MRI for Migraine}

Functional MRI ( $\mathrm{fMRI}$ ) is an imaging method that measures changes in the amount of oxidized hemoglobin associated with increased brain activity on the basis of blood oxygenation level [29]. $\mathrm{fMRI}$ can be broadly classified into task-based $\mathrm{fMRI}$, which examines activity during a given task, and resting-state $\mathrm{fMRI}$ ( $\mathrm{rs}-\mathrm{fMRI}$ ), which considers resting as a task. In task-based fMRI, the activation of the temporal pole, anterior cingulate cortex, somatosensory cortex, etc., is stronger in patients with migraine than in normal subjects. The temporal pole, anterior cingulate cortex, and somatosensory cortex are strongly activated in patients with migraine as compared to that in normal subjects; thus, suggesting changes in brain activity and dysfunction in patients with migraine [30].

Several studies have shown that the hypothalamic activity changes with the ictal/inter-ictal phase. Schulte et al. [31] reported migraine cycles for over 30 days by using MRI for one patient with migraine but without aura. Migraine attacks are classified as pre-ictal, ictal, post-ictal, and inter-ictal. At $24 \mathrm{~h}$ before onset, the hypothalamus and visual cortex were more activated than that in the interictal phase, and the middle pons was significantly activated in the ictal phase as compared to that in the inter-ictal phase. The functional connectivity (FC) between the hypothalamus and the spinal trigeminal nucleus was enhanced in the pre-ictal phase, while in the ictal phase, the FC between the hypothalamus and the dorsal rostral pons was significantly enhanced. FC is defined as the temporal coherence between different neurons, and because the connectivity is inferred by temporal correlations, it is also found between regions that differ from the actual anatomical connectivity [32].

In a study of a large number of patients, hypothalamic activation was observed at $48 \mathrm{~h}$ before the onset of attacks, and these activations were not present at $72 \sim 48 \mathrm{~h}$ before the onset of attacks. These results suggest that changes in brain function in patients with migraine occur in the hypothalamus and begin at $48 \mathrm{~h}$ before the onset of the attack [32]. Coppola et al. [33] reported that patients with migraine in the ictal phase showed higher activation in the medial prefrontal cortex (MPFC) and posterior cingulate cortex than healthy controls, and the FC between the MPFC and insula showed a negative correlation with attack intensity. In other words, the higher the intensity of the migraine attack, the lower is the connectivity. The insular cortex is related to various networks and is associated with multiple sensory, motor, and limbic systems. The findings of the authors suggest that the more painful the headache attack, the lower is the FC associated with the island, resulting in abnormal performance in various switching and other activities. Another study reported the connectivity between the inter-ictal phase and the ictal phase in patients with migraines without aura. Amin et al. [34] reported that the connectivity between the right thalamus and the left insula, the orbitofrontal cortex, was higher and that the connectivity between the right thalamus and the right somatosensory cortex was lower in migraine in the ictal phase as compared to that in migraine without aura in the inter-ictal phase. As mentioned above, migraine is a cyclical disease, and MRI findings vary depending on the phase in which the patient was imaged: pre-ictal, ictal, post-ictal, or inter-ictal; hence, it is important to confirm the findings with a headache diary or app. In addition, some studies used healthy subjects as the comparison group, while others used migraineurs with different cycles, such as those in inter-ictal and ictal periods; therefore, it is necessary to consider which comparison group is appropriate. Brain activation in patients with migraine is shown in Figure 3. 


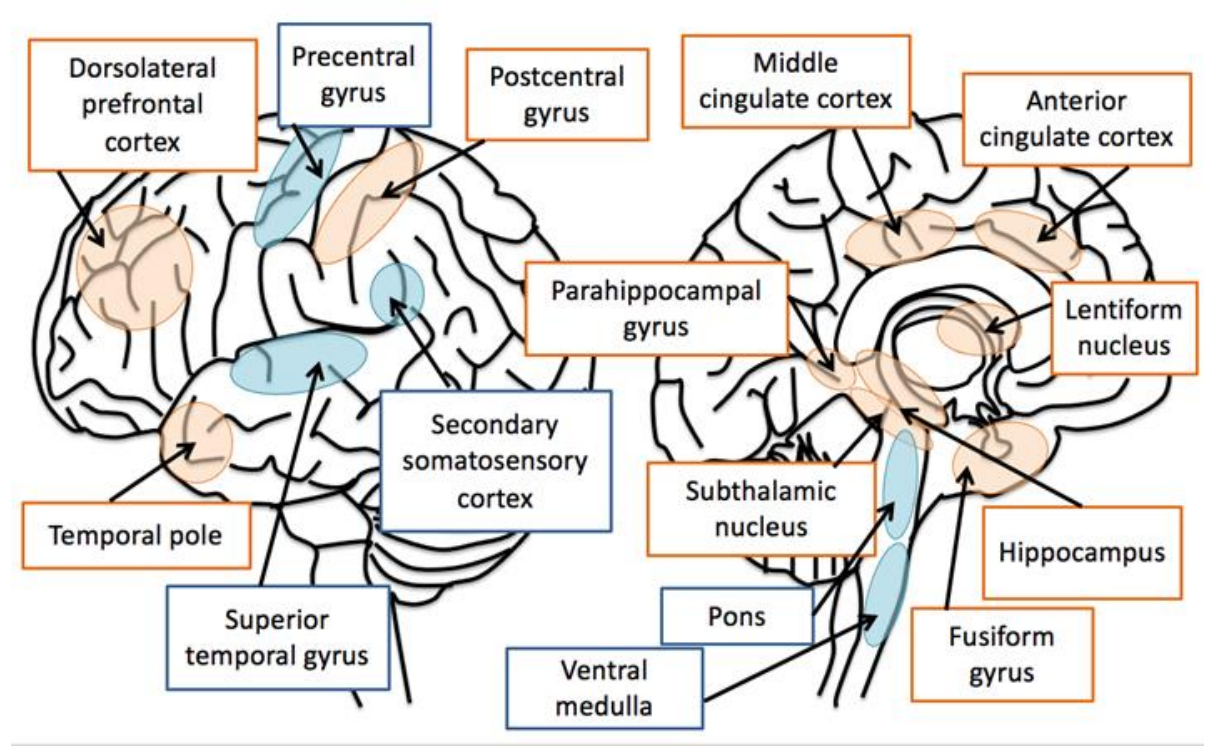

Figure 3 Pain-induced brain activations that differ in migraineurs versus healthy controls. Red regions show more activation in patients with migraines than in healthy controls. Blue regions show less activation in migraineurs than in healthy controls.

According to the International Classification of Headache, 3rd edition, migraine is classified as either episodic migraine (EM) or chronic migraine (CM) according to the number of attacks [35]. CM is defined as a headache that occurs at least 15 days per month for more than three months and at least eight days per month with migraine characteristics. In CM during the inter-ictal period, the number of years of $\mathrm{CM}$ and the FC between the left anterior insula-right periaqueductal gray, the right anterior insula-right mediodorsal thalamus, and the right anterior insula-right periaqueductal gray showed significantly positive correlations [36]. In other words, the longer the duration of chronicity, the higher is the FC associated with pain. In the whole-brain analysis, the association of the FC with the pain matrix was significantly stronger in CM than in EM [37]. Furthermore, the connectivity of the pain matrix to the hypothalamus was stronger in CM than in EM, while the connectivity to the dorsal raphe nucleus was significantly stronger in EM; this suggests that stronger connectivity of the pain matrix is a characteristic of chronic migraine.

\section{Conclusions}

Because of its non-invasive nature, MRI can be repeatedly performed. On the basis of this advantage of MRI, the pathophysiology of migraine has been investigated by using MRI. However, image findings are influenced not only by migraine alone. Age, sex, other comorbidities, mental status, acquisition parameters, and other variable conditions also influence MRI findings. Migraine phenotype, disease duration, medication, and other therapies also affect the results of MRI. By continuing active research on this topic, significant contributions are expected to be made to the diagnosis and therapy for migraines.

\section{Author Contributions}

YS, MS, SI all contributed to drafting and reviewing the manuscript. 


\section{Competing Interests}

The authors have declared that no competing interests exist.

\section{References}

1. Palm-Meinders IH, Hille K, Terwindt GM, Launer $L$, Konishi J, Moonen JM, et al. Structural brain changes in migraine. JAMA. 2012; 308: 1889-1896.

2. Fazekas F, Kleinert R, Offenbacher $H$, Schmidt R, Kleinert G, Payer F, et al. Pathologic correlates of incidental MRI white matter signal hyperintensities. Neurology. 1993; 43: 1683.

3. Webb ME, Amoozegar F, Harris AD. Magnetic resonance imaging in pediatric migraine. Can J Neurol Sci. 2019; 46: 653-665.

4. Faria V, Erpelding N, Lebel A, Johnson A, Wolff R, Fair D, et al. The migraine brain in transition: Girls vs boys. Pain. 2015; 156: 2212-2221.

5. Danielson NB, Guo JN, Blumenfeld H. The default mode network and altered consciousness in epilepsy. Behav Neurol. 2011; 24: 55-65.

6. Tu Y, Zeng F, Lan L, Li Z, Maleki N, Liu B, et al. An fMRI-based neural marker for migraine without aura. Neurology. 2020; 94: e741-e751.

7. Amin FM, Asghar MS, Hougaard A, Hansen AE, Larsen VA, Koning PJ, et al. Magnetic resonance angiography of intracranial and extracranial arteries in patients with spontaneous migraine without aura: A cross-sectional study. Lancet Neurol. 2013; 12: 454-461.

8. Arkink EB, Terwindt GM, Craen AJ, Konishi J, Grond J, Buchem MA, et al. Infratentorial microbleeds another sign of microangiopathy in migraine. Stroke. 2015; 46: 1987-1989.

9. Kurth T, Winter AC, Eliassen AH, Dushkes R, Mukamal KJ, Rimm EB, et al. Migraine and risk of cardiovascular disease in women: Prospective cohort study. BMJ. 2016; 353: i2610.

10. Monteith T, Gardener H, Rundek T, Dong C, Yoshita M, Elkind MS, et al. Migraine, white matter hyperintensities, and subclinical brain infarction in a diverse community: The northern Manhattan study. Stroke. 2014; 45: 1830-1832.

11. Bosemani T, Burton VJ, Felling RJ, Leigh R, Oakley C, Poretti A, et al. Pediatric hemiplegic migraine: Role of multiple MRI techniques in evaluation of reversible hypoperfusion. Cephalalgia. 2014; 34: 311-315.

12. Cadiot D, Longuet R, Bruneau B, Treguier C, Carsin-Vu A, Corouge I, et al. Magnetic resonance imaging in children presenting migraine with aura: Association of hypoperfusion detected by arterial spin labelling and vasospasm on MR angiography findings. Cephalalgia. 2018; 38: 949958.

13. Uetani H, Kitajima M, Sugahara T, Kikuchi H, Muto Y, Hirahara T, et al. Perfusion abnormality on three-dimensional arterial spin labeling with a $3 T$ MR system in pediatric and adolescent patients with migraine. J Neurol Sci. 2018; 395: 41-46

14. Lodi R, Montagna P, Soriani S, lotti S, Arnaldi C, Cortelli P, et al. Deficit of brain and skeletal muscle bioenergetics and low brain magnesium in juvenile migraine: An in vivo 31P magnetic resonance spectroscopy interictal study. Pediatr Res. 1997; 42: 866-871.

15. Barbiroli B, Montagna P, Cortelli P, Martinelli P, Sacquegna T, Zaniol P, et al. Complicated migraine studied by phosphorus magnetic resonance spectroscopy. Cephalalgia. 1990; 10: 263272. 
16. Assarzadegan F, Asgarzadeh S, Hatamabadi HR, Shahrami A, Tabatabaey A, Asgarzadeh M. Serum concentration of magnesium as an independent risk factor in migraine attacks: $A$ matched case-control study and review of the literature. Int Clin Psychopharmacol. 2016; 31: 287-292.

17. Ramadan NM, Halvorson H, Vande-Linde A, Levine SR, Helpern JA, Welch KM. Low brain magnesium in migraine. Headache. 1989; 29: 590-593.

18. McCarty MF. Magnesium taurate and fish oil for prevention of migraine. Med Hypotheses. 1996; 47: 461-466.

19. Altura BM, Altura BT, Carella A, Gebrewold A, Murakawa T, Nishio A. $\mathrm{Mg}^{2+}-\mathrm{Ca}^{2+}$ interaction in contractility of vascular smooth muscle: $\mathrm{Mg}^{2+}$ versus organic calcium channel blockers on myogenic tone and agonist-induced responsiveness of blood vessels. Can J Physiol Pharmacol. 1987; 65: 729-745.

20. Wang SJ, Lirng JF, Fuh JL, Chen JJ. Reduction in hypothalamic $1 \mathrm{H}-\mathrm{MRS}$ metabolite ratios in patients with cluster headache. J Neurol Neurosurg Psychiatry. 2006; 77: 622-625.

21. Coppola G, Tinelli E, Lepre C, lacovelli E, Lorenzo CD, Lorenzo GD, et al. Dynamic changes in thalamic microstructure of migraine without aura patients: A diffusion tensor magnetic resonance imaging study. Eur J Neurol. 2014; 21: 287-e13.

22. Planchuelo-Gómez Á, García-Azorín D, Guerrero ÁL, Aja-Fernández S, Rodríguez M, Luis-García R. White matter changes in chronic and episodic migraine: A diffusion tensor imaging study. J Headache Pain. 2020; 21: 1-15.

23. Rocca MA, Ceccarelli A, Falini A, Tortorella P, Colombo B, Pagani E, et al. Diffusion tensor magnetic resonance imaging at 3.0 tesla shows subtle cerebral grey matter abnormalities in patients with migraine. J Neurol Neurosurg Psychiatry. 2006; 77: 686-689.

24. Shibata Y. Recent developments in neuroimaging for the evaluation of migraines. Int J Radiol. 2015; $2: 15-16$.

25. Shibata $Y$, Ishiyama S, Matsushita A. White matter diffusion abnormalities in migraine and medication overuse headache: A 1.5-T tract-based spatial statistics study. Clin Neurol Neurosurg. 2018; 174: 167-173.

26. Szabó N, Kincses ZT, Párdutz Á, Tajti J, Szok D, Tuka B, et al. White matter microstructural alterations in migraine: A diffusion-weighted MRI study. PAIN. 2012; 153: 651-656.

27. Yuan K, Qin W, Liu P, Yu D, Zhao L, Dong M, et al. Reduced fractional anisotropy of corpus callosum modulates inter-hemispheric resting state functional connectivity in migraine patients without aura. PLoS One. 2012; 7: e45476.

28. Zhang $\mathrm{H}$, Schneider T, Wheeler-Kingshott CA, Alexander DC. NODDI: Practical in vivo neurite orientation dispersion and density imaging of the human brain. Neurolmage. 2012; 61: 10001016.

29. Fox MD, Raichle ME. Spontaneous fluctuations in brain activity observed with functional magnetic resonance imaging. Nat Rev Neurosci. 2007; 8: 700-711.

30. Schwedt TJ, Chiang C, Chong CD, Dodick D. Functional MRI of migraine. Lancet Neurol. 2015; 14: 81-91.

31. Schulte LH, Mehnert J, May A. Longitudinal neuroimaging over 30 days: Temporal characteristics of migraine. Ann Neurol. 2020; 87: 646-651.

32. Horwitz B. The elusive concept of brain connectivity. Neuroimage. 2003; 19: 466-470. 
33. Coppola G, Renzo AD, Tinelli E, Lorenzo CD, Scapeccia M, Parisi V, et al. Resting state connectivity between default mode network and insula encodes acute migraine headache. Cephalalgia. 2018; 38: 846-854.

34. Amin FM, Hougaard A, Magon S, Sprenger T, Wolfram F, Rostrup E et al. Altered thalamic connectivity during spontaneous attacks of migraine without aura: A resting-state fMRI study. Cephalalgia. 2018; 38: 1237-1244.

35. Headache Classification Committee. The international classification of headache disorders, 3rd ed. Cephalalgia. 2018; 38: 1-211.

36. Schwedt TJ, Schlaggar BL, Mar S, Nolan T, Coalson RS, Nardos B, et al. A typical resting-state functional connectivity of affective pain regions in chronic migraine. Headache. 2013; 53: 737 751.

37. Lee MJ, Park B, Cho S, Kim ST, Park H, Chung CS. Increased connectivity of pain matrix in chronic migraine: A resting-state functional MRI study. J Headache Pain. 2019; 20: 1-10.

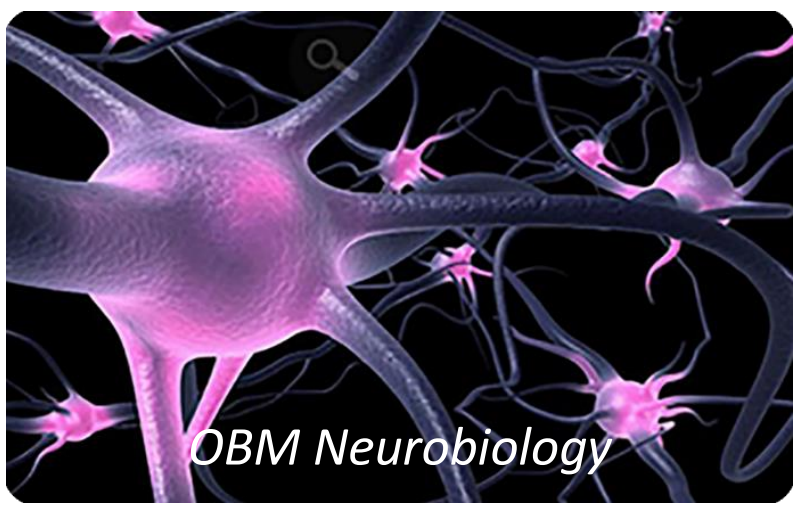

Enjoy OBM Neurobiology by:

1. Submitting a manuscript

2. Joining in volunteer reviewer bank

3. Joining Editorial Board

4. Guest editing a special issue

For more details, please visit:

http://www.lidsen.com/journals/neurobiology 